\title{
Efficient IoT-enabled Landslide Monitoring
}

\author{
Matthew Butler \\ Bournemouth University \\ Dr. Marios Angelopoulos \\ Bournemouth University \\ Doug Mahy \\ Poole, Dorset, UK \\ Poole, Dorset, UK \\ Net Sensors Ltd \\ Email: s4914562@bournemouth.ac.uk Email: mangelopoulos@bournemouth.ac.uk Email: doug.mahy@netsensors.co.uk
}

\begin{abstract}
Landslides pose significant socio-economic threats to areas whose geography favors them. Currently existing landslide monitoring methods and techniques are characterized by significant limitations both in technical terms (quality and frequency of data) and in terms of usability (high inferred costs, requirement of very high expertise). In this work we present an innovative landslide monitoring system that leverages state-of-the-art IoT technologies. The system consists of a set of autonomous sensing devices equipped with a sensor suit specifically tailored for monitoring landslides. The devices take sensory measurements at frequent intervals - while operating at a very low duty cycle - and transmit them over the SigFox network to a data server powered by ELK stack for curation and visualization. The system has been successfully deployed in a landslide site at Bournemouth, UK providing the local authorities with a new means of efficient and remote monitoring. The system follows a modular scalable architecture and has been proven to be highly reliable. As a result, there are plans for its use to be extended to other parts of the Bournemouth area as well as of the UK.
\end{abstract}

Index Terms-Internet of Things, SigFox, landslide

\section{INTRODUCTION}

The Internet of Things (IoT) is a term for describing an infrastructure for enabling advanced, ubiquitous services between connected physical and virtual things, based on evolving information and communication technologies in an Internet-like structure [1]. Things in IoT are described as objects embedded with sensors and the ability to communicate, resulting in networks that underpin new innovative business models and processes, whilst reducing the associated costs and risks of existing ones [2]. The techno-social ecosystem of IoT enables a cyber-physical convergence which lies at the heart of broader paradigm shifts, such as Industry 4.0 [3] and Circular Economy [4]. The financial impact of IoT is also profound; by 2025 more than 55 billion IoT devices will be deployed globally and around 15USD trillions will be invested in IoT in aggregate between 2017 and 2025 [5]. Investment areas already cover several sectors of economic activity and use-case verticals such as health care, security, transportation, critical infrastructure, manufacturing, etc [6]. However, the benefits of IoT are not confined only in reducing the costs and increasing the efficiency of already existing systems, but extend to enabling new innovative services that were not possible to be supported so far. These services not

978-1-5386-4980-0/19/\$31.00 @ 2019 IEEE only lead to increased yields but also inform the decision making of end-users.

Recent technological advancements and trends have accelerated the deployment and adoption of IoT networks and systems. The proliferation of Single Board Computers [7] has greatly reduced the CapEx and has increased the versatility of developing and deploying IoT systems. Advancements in wireless communication technologies have also been another important factor. Low power personal area network (LowPANs) protocols, such as IEEE802.15.4 and Bluetooth Low Energy (BLE) at the physical and MAC layers, and 6LoWPAN and 6TiSCH at the networking layers have enabled IoT systems in indoor environments such as smart buildings [8] and factories [9], [10]. On the other hand, wireless wide area networks for the IoT, such as LoRaWAN [11], SigFox [12] and NB-IoT [13] enable the deployment of IoT systems over broad geographic areas, thus allowing novel business models and decision making mechanisms for private companies as well as for local and national authorities.

Our Contribution. In this work we present an IoT system for efficient landslide monitoring that has been developed for the Bournemouth Borough Council (UK). The system consists of custom-made sensing devices, equipped with a tailored sensor suit for landslide monitoring, that are deployed in the area of interest. The devices transmit sensory data wirelessly over the SigFox network to a back-end server running an MQTT broker and the ELK stack for data curation and visualization. The system greatly improves to currently employed monitoring methods in terms of i) ease of deployment, scalability and cost efficiency - reduced capital and operating expenses; ii) enabling remote data collection; iii) providing data of high precision and granularity in near-real time (a new set of measurements every ten minutes); iv) ease and agility of use for the end-user (customizable dashboards, support of multiple devices).

Contrary to currently available state of the art solutions, the system we present in this work constitutes a cost-efficient, highly-reliable and scalable solution that is able to collect and report sensory data of very high precision in near-real time and in a continuous way. This performance is achieved by leveraging upon IoT technologies combined with powerful back-end solutions for big data curation and visualization. As such, this work nicely demonstrates the power of IoT not only in reducing CapEx and OpEx costs but also enabling the provision of novel services that previously were not feasible. 


\section{RELATED WORK ON LANDSLIDE MONITORING}

\section{A. Conventional Methods}

The term landslide refers to major soil displacements down a slope such as cliffs or mountains. Although, the primary driving force of a landslide is gravity, there are other contributing factors as well, such as soil composition, precipitation, variations of soil and air temperature and human activity. Landslides may have great adverse effects severely affecting human activity and lives, sometimes to the degree of natural disasters. Indicative examples are the 2010 Uganda landslide that caused 100 deaths and the 2011 Rio de Janeiro landslide that caused 610 deaths. In this context, landslide monitoring and prediction has been a hot research topic.

Currently available landslide monitoring techniques can coarsely be taxonomized in methods requiring physical access and methods of remote monitoring [14]. Topographic surveys and on-site visual inspection by experts is probably the most commonly employed method; however, this method is subject to ease of access and does not scale well with respect to area coverage. More accurate methods include the use of sophisticated equipment, such as terrestrial laser scanners [15], extensometers [16], tensiometers and inclinometers [17]. However, these methods require specific expertise and introduce high operating costs. Furthermore, these methods are also not easily scaled to cover big areas. Remote monitoring methods include the use of satellites, either in the form of GPS systems [18] or interferometry techniques via synthetic aperture radar (SAR) satellite images [19]. It is worth mentioning, that apart from the issues already mentioned, none of the aforementioned methods is able to provide data in real or near-real time. Also, some of these methods are not accessible to authorities of local communities either due to them requiring high expertise or due to inferred costs.

\section{B. Methods using Wireless Sensor Networks}

Wireless Sensor Networks (WSNs) are peer-to-peer adhoc networks consisting of small autonomous sensing devices (a.k.a. sensor motes) that are able to collaboratively carry out complex tasks. WSNs are a key enabling technology of IoT [20] and their paradigm has contributed a lot in developing core IoT technologies such as IEEE802.15.4 and 6LoWPAN. There is rich literature on WSNs being employed in several applications such as smart buildings [21], forest fire detection [22] and smart grid [23].

Regarding landslide monitoring with WSNs, the line of research presented in [24], [25] is probably the most notable one. The authors design a column that houses several sensors for detecting landslides. In particular, the sensor suite consists of:

- Dielectric Moisture Sensors: Measuring water content of the soil.

- Pore Pressure Piezometers: Measures groundwater pore pressure.

- Strain gauges: Measures movement of soil layers attached to the Deep Earth Probe (DEP).
- Tilt-meter: Measuring movement of soil layers in regards to creep, slow or sudden movements.

- Geophones: Measures vibrations caused during a landslide.

- Rain Gauges: Measures the effect of rainfall on a slope and therefore the ancillary effects such as pore pressure.

- Temperature Sensors: Physical properties of soil and water change with temperature, recorded every fifteen minutes.

The success of these devices was demonstrated by the early detection of a landslide in July 2009, providing validation for the authors' design during a heavy rainfall period in India's monsoon season. Criticisms of this system rely on the large physical form factor of the sensor columns (20 meters in length), their high energy consumption (the column relies upon a constant, wired power source which is backed by a power bank and a solar panel) and their high cost.

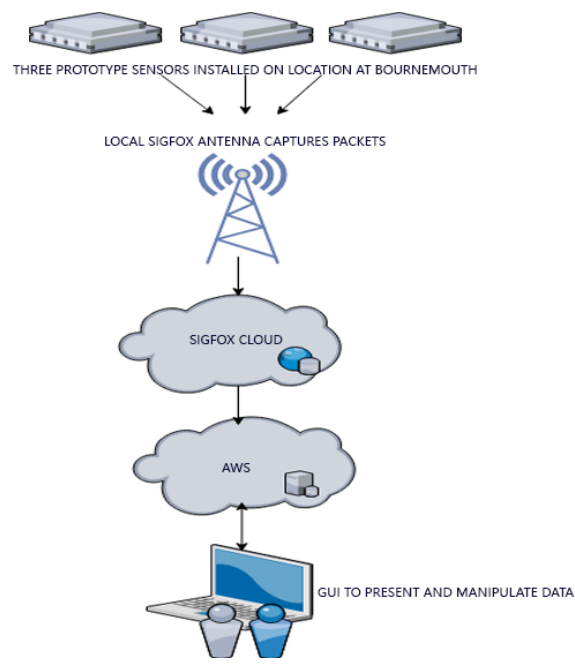

Fig. 1. High level architecture of the deployed IoT system for landslide monitoring

Another prototype network is presented in [26]. Authors present a novel system for landslide detection based on WSN sensor motes equipped with a bi-axis accelerometer. The motes communicate via Bluetooth to a transceiver where the accelerometer sensor data can be analysed. The system is evaluated using a small scale artificial slope fitted with sensor motes. Although this presents a cost-effective means to monitor landmass, the system presented features the use of Bluetooth - which has an effective range of 30 meters in line of sight - and high battery consumption that makes the system unsuitable for real-life deployment. Most importantly, the system needs to be calibrated for each individual site by pre-defining slope condition thresholds in order to reduce false-positive and false-negative detections.

\section{The Landslide Monitoring Iot Network}

Bournemouth is a coastal city located on the South Coast of the UK. Its landscape is characterized by several miles of coastline overseen by high cliffs, and neighbours the world 


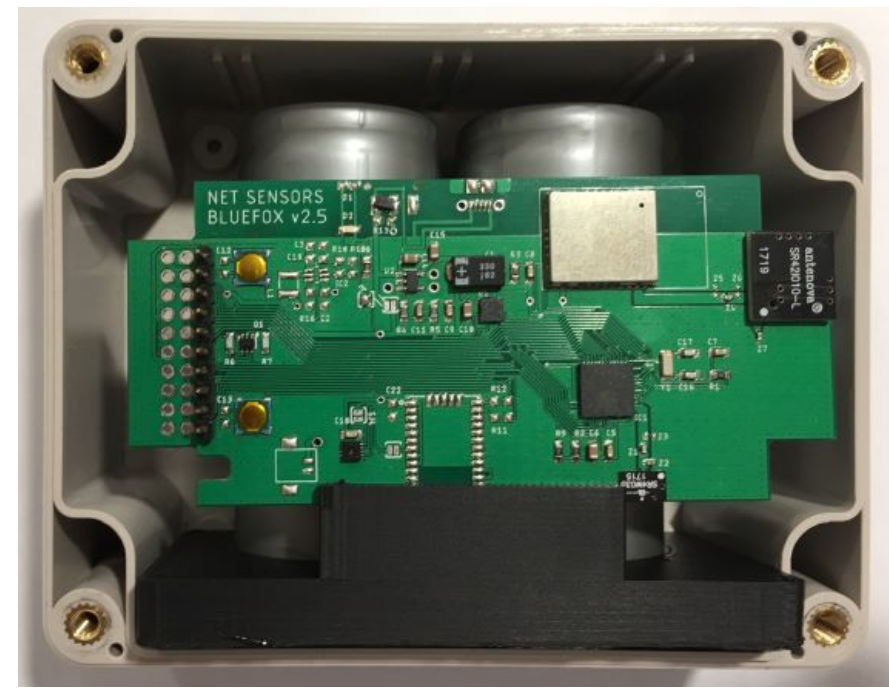

Fig. 2. One of the sensing motes in its casing. The device features among other, a dual microprocessor, SigFox connectivity, a 3-axis accelerometer, soil humidity and temperature sensors and is powered by two $4200 \mathrm{mAh}$ batteries (visible in the back of the device)

heritage Jurassic Coast. In April 2016 a major landslide took place that has demolished public access buildings and infrastructure (thankfully with no human casualties). So far, the total cost for the local council to clean-up and keep the surrounding areas safe rises to several million pounds, also including officers' time and external contractors for surveying and monitoring the landslide site.

Bournemouth is also one of the four focus areas in the UK for the promotion of IoT, in the context of the national programme Things Connected [27]. As such, in 2017 twenty seven SigFox access points were deployed in public buildings under permission of the Bournemouth Borough Council (BBC), providing a 100\% coverage for the surrounding areas. Later in the same year, in the context of Things Connected, $\mathrm{BBC}$ issued the challenge of developing an innovative landslide monitoring system with the following functional requirements:

- The system should be able to monitor the landslide independently of any vegetation or other infrastructure (e.g. coir nets) present

- The system should be able to provide high fidelity data with centimetre-scale accuracy

- The system should be able to allow remote monitoring of the landslide

- It would be desired for the data transmission to be wireless

- It would be desired for the data transmission to be (near-) real time

Several proposed designs were submitted, among others including the use of public-safety CCTV cameras to be used for capturing still images of the area of interest that would be then analysed, the use of laser-scanners fitted on flying drones and the use of satellite images. Bournemouth University and

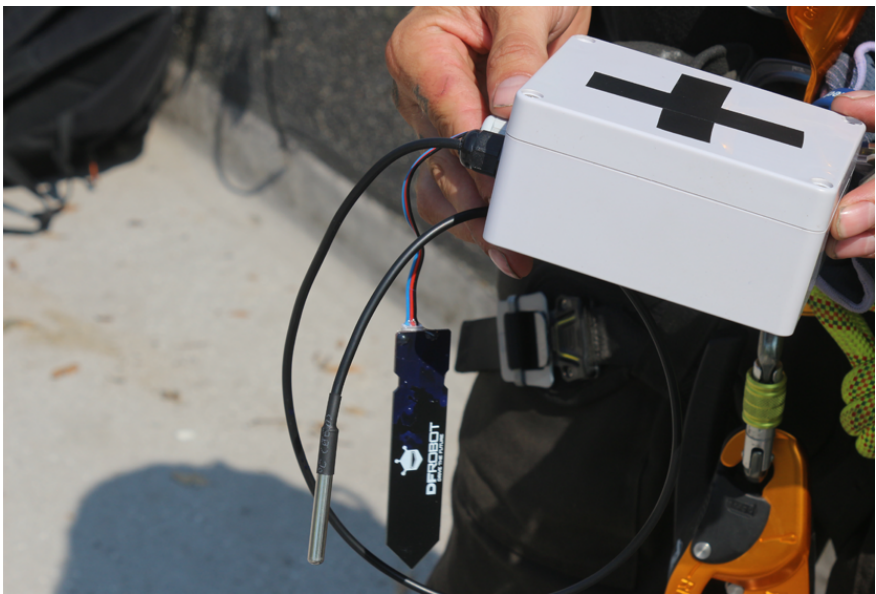

Fig. 3. The sensing device in its final form prior to being deployed at the landslide site. The soil humidity and temperature sensors are visible, waterproofed via silicon glands. Installation was undertaken by specialists rope technicians and geotechnical engineers.

Net Sensors Ltd proposed the design and development of an IoT-based solution that would leverage the recently deployed SigFox network 1 . The system would consist of custom-made sensor motes optimized for landslide monitoring that would be deployed in the area of interest. Sensory data would be transmitted in frequent intervals over the SigFox network to a back-end server which would store and visualize the data while supporting sophisticated machine-learning and data analytics algorithms for anomalies detection indicating an increased probability of a landslide to take place. Depending on the achieved performance, the system would be able to act both as a continuous monitoring systems as well as an early warning system for landslides.

The proposal was preferred over the rest due to the reduced inferred costs, ease of use and fidelity of data it could deliver. In the following, we provide a detailed presentation of the developed system which is currently (late 2018) deployed and operating.

\section{A. Architecture and instrumentation}

The sensor motes developed for the needs of the system are based on the BlueFox v2.7 platform, provided by Net Sensors Ltd. The boards feature two low-power microprocessors, a SigFox modem, a 3-axis digital output accelerometer, humidity and temperature sensors. The sensing capabilities of the board were extended by means of a DS18B20 Waterproof Digital Thermometer and the Analog Capacitive Soil Moisture Sensor V1.2 by DFRobot. Finally, the motes were powered by two IFR32650H 4200mah batteries each.

Since the motes were to be deployed in an outdoor environment exposed to weather conditions and other hazards (seagulls and rodents have been proven to pose great threats for any type of equipment), particular care was taken to protect the motes. They were waterproofed by the application of an acrylic conformal coating and an epoxy resin to protect the circuit boards of the capacitive sensor and they were encased 


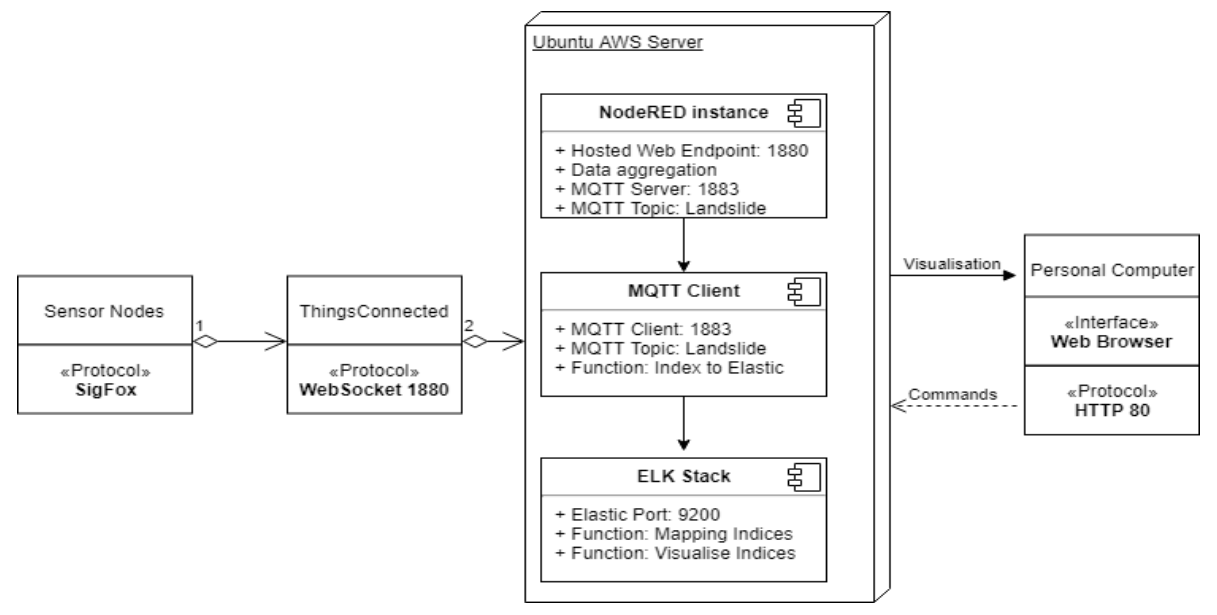

Fig. 4. Detailed architecture of the system depicting the various functional components and their inter-connectivity.

in industrial graded enclosure casings. The boards needed to be firmly attached to the casings so us not to affect the accuracy of the accelerometer readings; at the same time the attachment should not be permanent for development purposes. For this purpose a customized base was 3D printed. Figure 2 depicts one of the boards in its casing while figure 3 depicts the device in its final form.

The devices are organized in a one-hop star topology, each one connecting individually to a SigFox antennae located within proximity. The devices are partially buried at the landslide site, with the external soil sensors buried completely under ground. The devices operate on a low duty cycle, waking every 10 minutes to take and transmit sensory readings, then returning back to the deep sleep mode. With this scheme and the two $4200 \mathrm{mAh}$ batteries that each device is equipped with, the expectant longevity of the system is estimated at circa two years of continuous operation.

\section{B. SigFox packet structure}

TABLE I

SigFox PACKET STRUCTURE

\begin{tabular}{|c|c|c|c|c|c|}
\hline $\begin{array}{c}\text { Preamble } \\
(4 \text { bytes })\end{array}$ & $\begin{array}{c}\text { Frame Sync } \\
(2 \text { bytes })\end{array}$ & $\begin{array}{c}\text { Device ID } \\
(4 \text { bytes })\end{array}$ & $\begin{array}{c}\text { Payload } \\
(0-12 \text { bytes })\end{array}$ & $\begin{array}{c}\text { Auth } \\
(\text { var })\end{array}$ & $\begin{array}{c}\text { FCS } \\
(2 \text { bytes })\end{array}$ \\
\hline
\end{tabular}

TABLE II

PAYLOAD Allocation

\begin{tabular}{|c|c|c|c|c|c|c|}
\hline \multicolumn{7}{|c|}{ Payload (12 Bytes) } \\
\hline Bytes 1-2 & Bytes 3-4 & Bytes 5-6 & Bytes 7-8 & Bytes 9-10 & Byte 11 & Byte 12 \\
X-axis & Y-axis & Z-axis & Soil H & Soil T & Battery Level & Software Version \\
\hline
\end{tabular}

The SigFox packet structure allows for a maximum of 12 bytes, containing the data required to perform the analysis. The majority of the payload is allocated to the built-in accelerometer alongside the external temperature and soil moisture probes. Two bytes are reserved for the battery level and software version. The network allows for 140 messages per day to be delivered, although this is subject to the subscription model - alongside payload size - available to the users.

\section{NodeRED}

Node-RED is a flow based development tool developed by IBM for connecting hardware devices and APIs towards the IoT paradigm [28]. It is implemented in JavaScript utilizing the Node.js framework, establishing a data-flow driven design tool that consists of JSON (Javascript Object Notation) data generated internally and externally from the application. NodeRED was employed to provide a means of establishing a modular framework for future extensions, and providing the means to export the data with ease towards the Elastic Stack.

\section{MQTT Server and Client}

Message Queuing Telemetry Transport (MQTT) is a lightweight publish and subscribe protocol for IoT and resource-constrained devices [29]. MQTT was chosen in similar regards to providing a modular system design, by publishing topics, such as landslide data, and subscribing to these so that they may be logged and sorted within the Elastic Stack. The MQTT client is featured as part of a Python script, which specifies which topics to subscribe to, and converts the timestamp and JSON packet into a searchable variable within Kibana.

\section{E. Elastic Stack}

The Elastic Stack or ELK stack is the terminology to define three open source projects, namely ElasticSearch, Logstash and Kibana. ElasticSearch - which is based off of Apache's Lucene Project - is a distributed, RESTFUL search and analytical engine as JSON over HTTP, and excels at indexing large amounts of text. Logstash manages events and logs, collecting them and parsing them for storage and later usage with the two complimentary technologies. Kibana is data visualization and exploration tool that acts as a dashboard for the stack. ELK has a low requirement for usability, making log analysis an almost trivial process evidenced by installing their own data-sets.

\section{EVALUATION AND VALIDATION}

Testing was performed at the landslide site to ensure successful communication between the nodes and the SigFox 


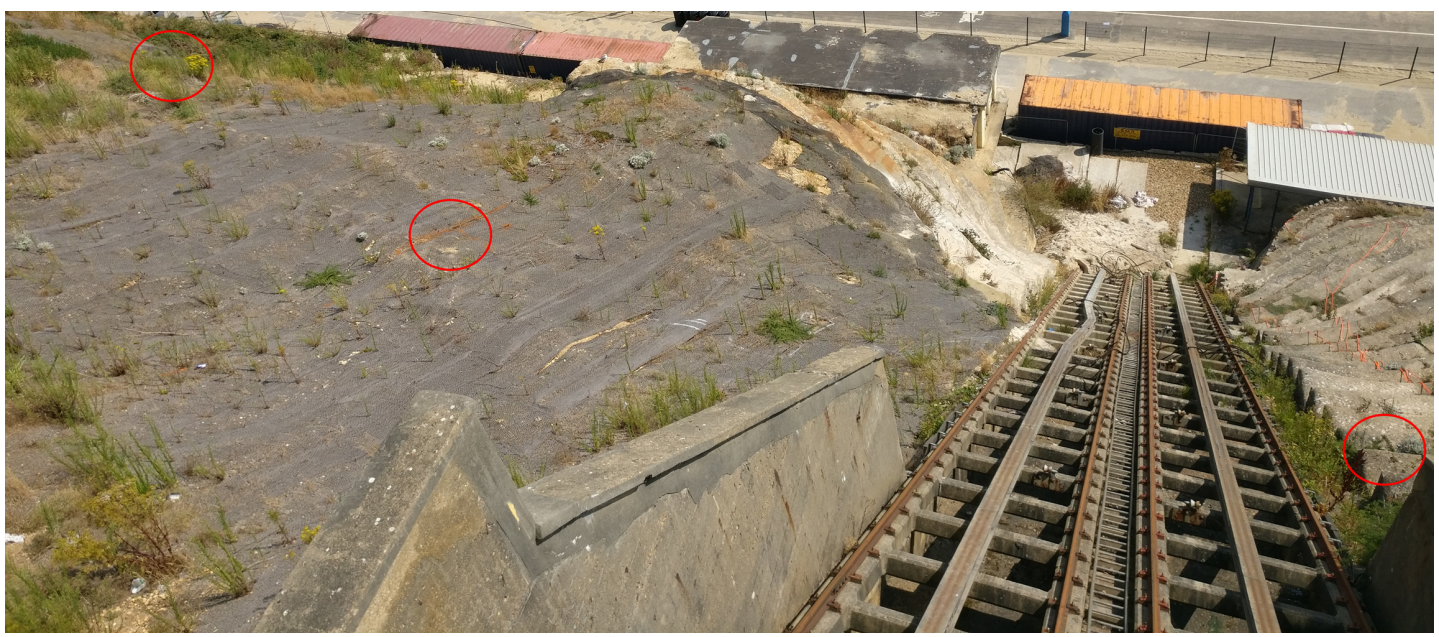

Fig. 5. The landslide area where the IoT-enabled monitoring system has been deployed. The landslide has taken place in the area on the left of the rails. At the top of the picture, the damaged public access buildings are visible, as well as the damage inflicted on the rail of the rail-wagon (used to facilitate public access to the beach). The red circles denote the positions of the installed sensing devices.

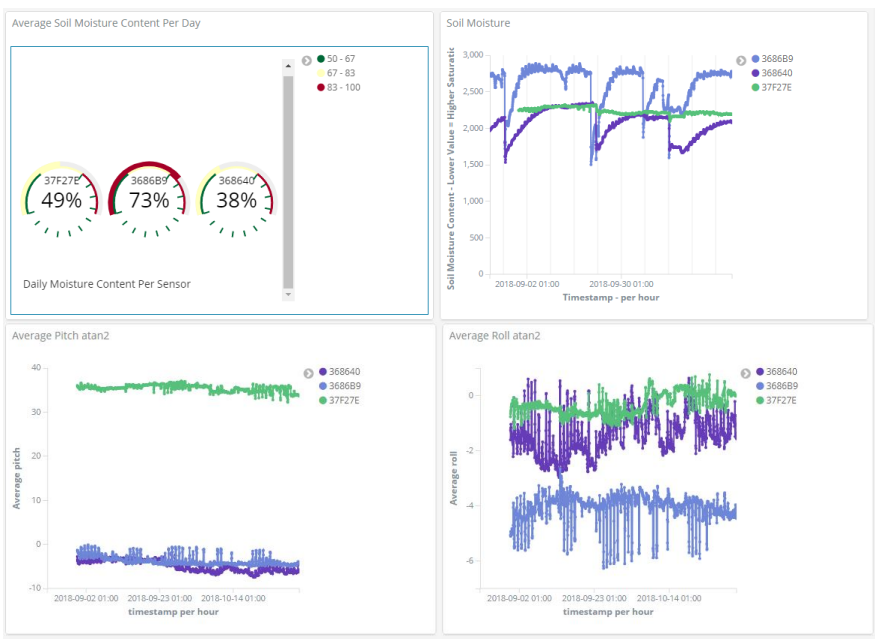

Fig. 6. Snapshots from the Kibana dashboard (not all tiles are depicted).

gateway. Three prototype devices were installed in locations of interest specified by Bournemouth Borough Council for the purposes of monitoring, with two devices completely buried, and one device partially-buried. Transmission was successful with the devices being buried up to a depth of 10 inches for the purposes of testing connectivity with the local SigFox access point. Transmitted packets were forwarded towards the Things Connected SigFox cloud, where a callback function forwards incoming traffic to the AWS server hosting the Elastic Stack.

With a Quality of Service set to $100 \%$ for the MQTT server, messages are guaranteed to arrive at least once after being received at the Node-RED server. Duplicate messages - in the event of - are dismissed when indexed into ElasticSearch.

Kibana powers the visualizations of the data in corresponding dashboards 6 by reporting the contents of the packets within an easily searchable GUI (Graphical User Interface). Here the dashboard acts as the centre page for the visualiza- tions, and gives Bournemouth Borough Council services the opportunity to explore in detail each device's reported data.

Since the installation (6 months in the time of writing), there has been zero loss of data during transmissions, except when maintenance has been needed and required the services to be stopped. During peaks of rainfall, the two buried devices correctly reported changes within soil moisture, temperature, and recorded movement impacted by the soil density. The third device recorded minimal changes within soil moisture which was to be expected, as with it's partial burial within the soil, its sensors are exposed to the elements.

It is worth noting that the modular design of the system consists of operational modules that are loosely coupled. This allows for the system to be easily scaled in terms of number of sensing motes and therefore of area coverage.

\section{CONCLUSION}

Landslides pose significant socio-economic threats to areas whose geography favours them. Currently existing landslide monitoring methods and techniques are characterized by significant limitations both in technical terms (quality and frequency of data) and in terms of applicability (high inferred costs, requirement of specific expertise). In this work we presented an innovative landslide monitoring system that leverages state-of-the-art IoT technologies. The system is highly reliable and scalable while its deployment and operation introduces significantly reduced costs when compared to existing alternatives.

The system has been successfully deployed providing the local Bournemouth Borough Council with a new means of monitoring a landslide site efficiently. There are plans for the system to be expanded to cover the entire coastal front of Bournemouth as well as other areas of the broader Dorset area. Discussions are in place with the British Geological Survey to investigate the introduction of the system to other areas of the UK as well. 
This work nicely demonstrates the profound impact of IoT not only in terms of reducing the costs of existing services but also in terms of enabling new innovative ones.

\section{REFERENCES}

[1] L. Atzori, A. Iera, and G. Morabito, "The internet of things: A survey," Computer networks, vol. 54, no. 15, pp. 2787-2805, 2010.

[2] M. Chui, M. Löffler, and R. Roberts, "The internet of things," McKinsey Quarterly, vol. 2, no. 2010, pp. 1-9, 2010.

[3] H. Lasi, P. Fettke, H.-G. Kemper, T. Feld, and M. Hoffmann, "Industry 4.0," Business \& Information Systems Engineering, vol. 6, no. 4, pp. 239-242, 2014.

[4] P. Ghisellini, C. Cialani, and S. Ulgiati, "A review on circular economy: the expected transition to a balanced interplay of environmental and economic systems," Journal of Cleaner production, vol. 114, pp. 1132, 2016.

[5] Statista. (2018) Size of the internet of things market worldwide in 2014 and 2020, by industry (in billion u.s. dollars). [Online]. Available: https://www.statista.com/statistics/512673/worldwideinternet-of-things-market/

[6] I. F. Akyildiz, W. Su, Y. Sankarasubramaniam, and E. Cayirci, "Wireless sensor networks: a survey," Computer networks, vol. 38, no. 4, pp. 393 422, 2002.

[7] S. J. Johnston, M. Apetroaie-Cristea, M. Scott, and S. J. Cox, "Applicability of commodity, low cost, single board computers for internet of things devices," in 2016 IEEE 3rd World Forum on Internet of Things (WF-IoT). IEEE, 2016, pp. 141-146.

[8] C. M. Angelopoulos, O. Evangelatos, S. Nikoletseas, T. P. Raptis, J. D. Rolim, and K. Veroutis, "A user-enabled testbed architecture with mobile crowdsensing support for smart, green buildings," in Communications (ICC), 2015 IEEE International Conference on. IEEE, 2015, pp. 573578.

[9] T. Raptis, A. Passarella, and M. Conti, "Performance analysis of latencyaware data management in industrial iot networks," Sensors, vol. 18, no. 8, p. 2611, 2018.

[10] T. P. Raptis, A. Passarella, and M. Conti, "Maximizing industrial iot network lifetime under latency constraints through edge data distribution," in 2018 IEEE Industrial Cyber-Physical Systems (ICPS). IEEE, 2018, pp. 708-713.

[11] F. Adelantado, X. Vilajosana, P. Tuset-Peiro, B. Martinez, J. MeliaSegui, and T. Watteyne, "Understanding the limits of lorawan," IEEE Communications magazine, vol. 55, no. 9, pp. 34-40, 2017.

[12] J. C. Zuniga and B. Ponsard, "Sigfox system description," LPWAN@ IETF97, Nov. 14th, 2016.

[13] R. Ratasuk, B. Vejlgaard, N. Mangalvedhe, and A. Ghosh, "Nb-iot system for $\mathrm{m} 2 \mathrm{~m}$ communication," in Wireless Communications and Networking Conference (WCNC), 2016 IEEE. IEEE, 2016, pp. 1-5.

[14] A. K. Turner and G. Jayaprakash, Landslides: investigation and mitigation. chapter 1-introduction. National Academies Press, 1996, no. 247.
[15] G. Bitelli, M. Dubbini, and A. Zanutta, "Terrestrial laser scanning and digital photogrammetry techniques to monitor landslide bodies," International Archives of Photogrammetry, Remote Sensing and Spatial Information Sciences, vol. 35, no. B5, pp. 246-251, 2004

[16] N. D. Rose and O. Hungr, "Forecasting potential slope failure in open pit mines-contingency planning and remediation," International Journal of Rock Mechanics and Mining Sciences, vol. 44, pp. 308-320, 2007.

[17] X. Tu, A. Kwong, F. Dai, L. Tham, and H. Min, "Field monitoring of rainfall infiltration in a loess slope and analysis of failure mechanism of rainfall-induced landslides," Engineering Geology, vol. 105, no. 1-2, pp. 134-150, 2009.

[18] J. A. Gili, J. Corominas, and J. Rius, "Using global positioning system techniques in landslide monitoring," Engineering geology, vol. 55, no. 3, pp. 167-192, 2000.

[19] L. Noferini, M. Pieraccini, D. Mecatti, G. Macaluso, C. Atzeni, M. Mantovani, G. Marcato, A. Pasuto, S. Silvano, and F. Tagliavini, "Using gbsar technique to monitor slow moving landslide," Engineering Geology, vol. 95, no. 3-4, pp. 88-98, 2007.

[20] S. Yinbiao, K. Lee, P. Lanctot, F. Jianbin, H. Hao, B. Chow, and J.-P. Desbenoit, "Internet of things: wireless sensor networks," White Paper, International Electrotechnical Commission, http://www. iec. ch, p. 11, 2014.

[21] C. M. Angelopoulos, G. Filios, S. Nikoletseas, D. Patroumpa, T. P. Raptis, and K. Veroutis, "A holistic ipv6 test-bed for smart, green buildings," in Communications (ICC), 2013 IEEE International Conference on. IEEE, 2013, pp. 6050-6054.

[22] A. Herutomo, M. Abdurohman, N. A. Suwastika, S. Prabowo, and C. W. Wijiutomo, "Forest fire detection system reliability test using wireless sensor network and openmtc communication platform," in Information and Communication Technology (ICoICT), 2015 3rd International Conference on. IEEE, 2015, pp. 87-91.

[23] W. Gans, A. Alberini, and A. Longo, "Smart meter devices and the effect of feedback on residential electricity consumption: Evidence from a natural experiment in northern ireland," Energy Economics, vol. 36, pp. 729-743, 2013.

[24] M. V. Ramesh, "Real-time wireless sensor network for landslide detection," in Sensor Technologies and Applications, 2009. SENSORCOMM'09. Third International Conference on. IEEE, 2009, pp. 405409.

[25] — , "Design, development, and deployment of a wireless sensor network for detection of landslides," Ad Hoc Networks, vol. 13, pp. 2-18, 2014

[26] H. W. Kim, "Development of wireless sensor node for landslide detection," Proceedings of the Asia-Pacific Advanced Network, vol. 42, pp. 56-60, 2016.

[27] ThingsConnected, "What is ThingsConnected?" [online]. Available From: https://www.thingsconnected.net/ [Accessed 14 June 2018], 2018.

[28] M. Blackstock and R. Lea, "Toward a distributed data flow platform for the web of things (distributed node-red)," in Proceedings of the 5th International Workshop on Web of Things. ACM, 2014, pp. 34-39.

[29] U. Hunkeler, H. L. Truong, and A. Stanford-Clark, "Mqtt-s-a publish/subscribe protocol for wireless sensor networks," in Communication systems software and middleware and workshops, 2008. comsware 2008. 3rd international conference on. IEEE, 2008, pp. 791-798. 